\title{
An Optical Search for Dwarf Novae in M22
}

\author{
A.P. Hourihane ${ }^{1}$, P.J. Callanan ${ }^{1}$, and A.M. $\mathrm{Cool}^{2}$ \\ ${ }^{1}$ Department of Physics, University College Cork, Cork, Ireland. \\ email: a.hourihane@ucc.ie,paulc@ucc.ie \\ ${ }^{2}$ Department of Physics and Astronomy, San Francisco State University, \\ 1600 Holloway Avenue, San Francisco, CA 94132, USA \\ email: cool@sfsu.edu
}

\begin{abstract}
We report on the results of our 2004 monitoring program of M22, designed to search for CVs undergoing dwarf nova (DN) eruptions. We wish to compare these globular cluster systems to similar ones in the field and determine if their different formation mechanisms result in different properties. We present a light curve for a CV candidate that went into outburst during May. Data were reduced with the ISIS image subtraction routine. Our ground-based results are consistent with previous $H S T$ measurements, and confirm the DN nature of the outburst. We also report on further attempts to apply the ISIS software to look for additional outbursts of this object during 2004 as well as to identify other variable candidates in the core of M22.
\end{abstract}

Keywords. stars: dwarf novae, techniques: photometric

\section{Introduction}

Compact binary systems - comprising a neutron star, black hole or white dwarf primary accreting from a low-mass companion - in globular clusters are an important target for observations. The process of their formation, unlike primordial field binaries, is thought to be three-body collisions and tidal capture (e.g., Hut \& Verbunt, 1983), leading in general to very close binaries, with perhaps different properties relative to their counterparts in the field.

So far, for example, relatively few DN outbursts of globular cluster CVs have been observed (e.g., Shara et al. 1996, Ó Tuairisg et al. 2003) compared to the predicted populations of such systems and it is of significant interest to determine if this is due to an underlying difference in the nature of the cluster CVs compared with field CVs.

Furthermore, the presence of HeII emission lines in the HST spectra of these systems suggests a possible magnetic nature (Grindlay et al. 1995).

To characterise these systems we present results from a long-term monitoring program of the globular cluster M22 during which a DN outburst of a cluster CV was observed.

A $\sim 3$ magnitude brightening of the same source was originally interpreted by Sahu et al. (2001) as a gravitational microlensing of a background bulge star by a low mass object in the globular cluster M22. Subsequent proper motion studies using HST archival data revealed cluster membership for the object, and this combined with its $\mathrm{H} \alpha$ brightness and outburst characteristics led Anderson et al. (2003) to conclude the object was in fact a cluster CV, CV1. Bond, et al. (2005) recorded two further similar outbursts between 2001 and 2004 as part of a MOA microlensing survey, effectively confirming the conclusions of Anderson et al. (2003). 


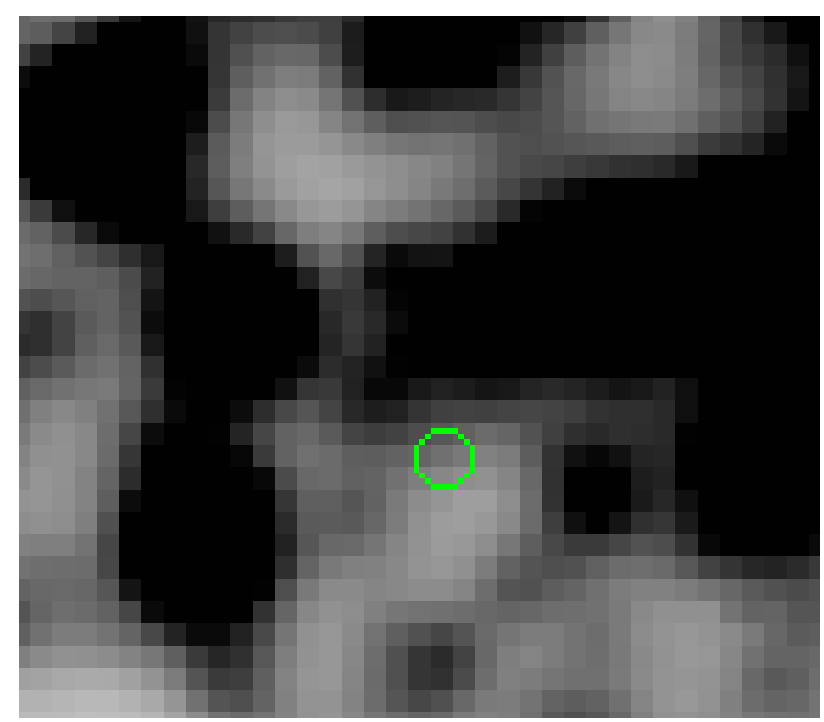

Figure 1. One of our $V$-band images showing the location of the CV during outburst. Its position is $R . A .=18^{\circ} 36^{\prime} 24^{\prime \prime} .66$, decl. $=-23^{\circ} 54^{\prime} 35^{\prime \prime} .5$ (J2000; Sahu et al. 2001)

\section{Data Analysis}

We obtained observations from the Cerro Tololo Inter-American Observatory (CTIO) 1.3-m telescope in Chile. The dataset consists of optical $V$-band images covering the period of March - November 2004. The pointing is centred on the core of globular cluster M22 (NGC 6656) and the images had a $6^{\prime} \times 6^{\prime}$ field of view. The sampling was approximately two 300s exposures every second night while the source was in quiescence and every night for the May period when it was observed to go into outburst. Photometry was performed with the ISIS (Alard \& Lupton 1998, Alard 2000) image-subtraction software. All the images were inspected for quality of seeing, and those in which it was found to be better than $1^{\prime \prime} .9$ were interpolated to the same reference grid, convolved to the same seeing and then subtracted from a reference image (a stack of approximately $10 \%$ of the best-quality images). A composite image of the subtracted frames was then created, showing all detected variables. Despite many spurious detections due to saturated stars, the $\mathrm{CV}$ is visible close to the centre of this image. This shows the remarkable power of ISIS when dealing with variables in crowded fields, as the CV is hardly visible in our images (Figure 1).

\section{Results}

Due to extreme crowding in the cluster core it was not possible to perform absolute photometry on the CV to transform the light curve (Figure 2) to standard magnitudes, therefore it is plotted in units of differential flux (that is the flux difference between each image and the reference frame, where the CV is in quiescence). There is a high degree of variability in the quiescent light curve which seems to be real - better sampling, and perhaps seeing, is needed to help clarify this point. The May outburst is also clearly visible with a timescale of $\sim 15$ days and $\mathrm{M}_{V} \sim 3.5$. There also appears to be a later, smaller-amplitude outburst in October. Only one sampling point is available but the duration can be constrained to a maximum of six days.

The amplitude and duration of the observed May 2004 outburst are consistent with 


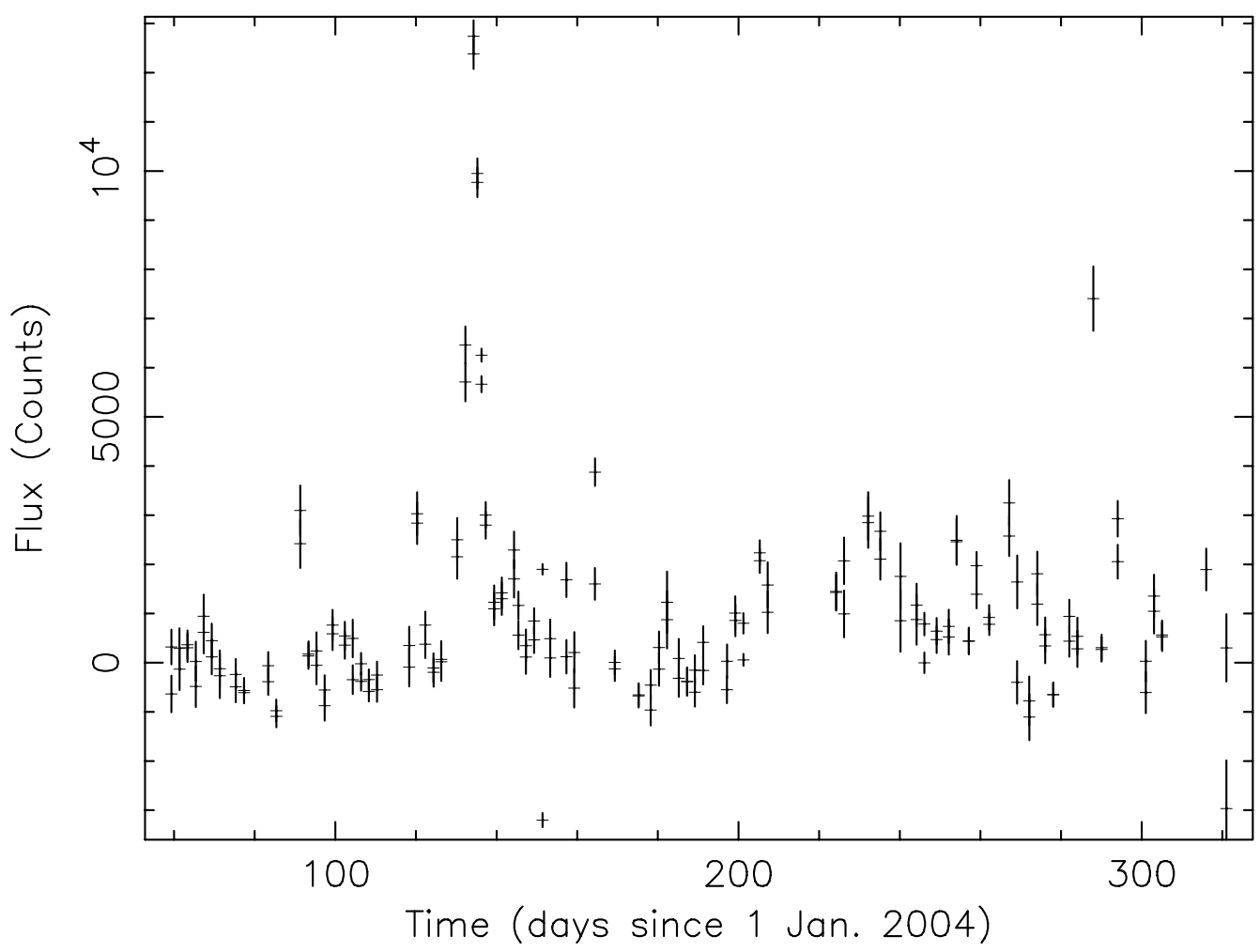

Figure 2. The ISIS differential flux light curve for the DN eruption of the M22 CV.

the previous outbursts documented by Sahu et al. (2001) and Bond, et al. (2005), again confirming the CV nature of the source proposed by Anderson et al. (2003).

\section{Discussion}

Our observations show a CV that underwent a DN eruption of $\mathrm{M}_{V} \sim 3.5$ in May 2004 and a probable later outburst of $\mathrm{M}_{V} \sim 2.5$ in October. The same system underwent at least three outburst episodes previous to this during the 1999-2004 period (Sahu et al, 2001; Bond et al, 2005). Therefore the outburst recurrence rate appears to be at least once every 1-2 years and perhaps more often than this.

Webb et al. (2004) observed $\mathrm{a} \sim 1 \mathrm{keV}$ absorption feature with XMM-Newton in an $\mathrm{X}$-ray source with an error circle of radius $5^{\prime \prime}$ enclosing the (optical) CV position. This feature may be interpreted as cyclotron resonance implying an intermediate polar (IP) - a subclass of dwarf novae with a weakly magnetised white dwarf primary - nature for the $\mathrm{CV}$.

The recurrence timescale and outburst amplitudes are consistent with a 'normal' dwarf nova or an IP. Strongly magnetised CVs are not observed to undergo outburst, however, in the more weakly magnetic IPs, even though outbursts starting at the inner disk edge are predicted to be rare, those starting at the outer edge are as frequent as in nonmagnetic systems, therefore small DN eruptions may not be uncommon amongst these systems (Angelini \& Verbunt 1989). 


\section{Conclusions}

For $C V 1$ in M22 we observed a $\sim 15$-day outburst of amplitude $\mathrm{M}_{V} \sim 3.5$ in May 2004 and a probable later outburst of $\mathrm{M}_{V} \sim 2.5$ in October of maximum duration six days. There is a possibility that this system is an IP but due to the length of the outbursts combined with a possible bimodality of outburst duration we believe it is more likely to be a non-magnetic dwarf nova. The next step is to search for more lower-amplitude shorter timescale outbursts from $C V 1$, the like of which may have been missed in the past, and from other CVs in globular clusters. For $C V 1$ these would confirm a bimodality of outburst duration and alone, for other CVs, would indicate a likely IP nature.

\section{References}

Alard, C. 2000, A\&AS 144, 363

Alard, C. \& Lupton, R.H. 1998, ApJ 503, 325

Anderson, J., Cool, A.M. \& King, I.R. 2003, ApJ 597, 137

Angelini, L. \& Verbunt, F. 1989, MNRAS 238, 697

Bond, I.A., Abe, F., Eguchi, S., Furuta, Y., Hearnshaw, J.B., Kamiya, K., Kilmartin, P.M., Kurata, Y., Masuda, K., Matsubara, Y., and 11 coauthors 2005, ApJ, 620, 103

Grindlay, J.E., Cool, A.M., Callanan, P.J., Bailyn, C.D., Cohn, H.N. \& Lugger, P.M. 1995, ApJ 455,47

Hut, P. \& Verbunt, F. 1983, Nature 301, 587

Ó Tuairisg, S., Butler, R.F., Shearer, A., Redfern, R.M., Butler, D. \& Penny, A. 2003, MNRAS 345,960

Sahu, K.C., Casertano, S., Livio, M., Gilliland, R.L., Panagia, N., Albrow, M.D. \& Potter, M. 2001, Nature 411, 1022

Shara, M., Bergeron, L., Gilliland, R., Saha, A. \& Petro, L. 1996, ApJ 471, 804

Webb, N.A., Serre, D., Gendre, B., Barret, D., Lasota, J.-P. \& Rizzi, L. 2004, A\&A, 424, 133 